\title{
Pratiques
}

Linguistique, littérature, didactique

\section{Intérêt et limites de la notion de dispositif}

L'exemple d'un dispositif d'enseignement de l'orthographe

Value and limits of the French concept of "dispositif". The example of a spelling intervention

Jacques Crinon et Caroline Viriot-Goeldel

\section{(2) OpenEdition}

Journals

\section{Édition électronique}

URL : https://journals.openedition.org/pratiques/10198

DOI : 10.4000/pratiques.10198

ISSN : 2425-2042

\section{Éditeur}

Centre de recherche sur les médiations (CREM)

\section{Référence électronique}

Jacques Crinon et Caroline Viriot-Goeldel, «Intérêt et limites de la notion de dispositif », Pratiques [En ligne], 189-190 | 2021, mis en ligne le 09 juillet 2021, consulté le 23 juillet 2021. URL : http:// journals.openedition.org/pratiques/10198; DOI : https://doi.org/10.4000/pratiques.10198

Ce document a été généré automatiquement le 23 juillet 2021.

(c) Tous droits réservés 


\title{
Intérêt et limites de la notion de dispositif
}

\author{
L'exemple d'un dispositif d'enseignement de l'orthographe \\ Value and limits of the French concept of "dispositif". The example of a spelling \\ intervention
}

Jacques Crinon et Caroline Viriot-Goeldel

\section{Qu'est-ce qu'un dispositif?}

1 Le succès actuel du terme tient probablement à deux influences, qui ont aussi contribué à définir certains traits du concept: l'emprunt au domaine technique (on parle par exemple couramment de " dispositifs médicaux ») et son emploi par M. Foucault (1977) qui, étudiant l'asile ou la prison, propose d'appeler dispositif un réseau qui relie différents éléments hétérogènes, "des discours, des institutions, des aménagements architecturaux, des décisions règlementaires, des lois, des mesures administratives, des énoncés scientifiques, des propositions philosophiques, morales, philanthropiques, bref : du dit, aussi bien que du non-dit » (p. 299), ensemble qui permet de contrôler les corps et les esprits.

2 Nous retiendrons donc d'abord le rapport entre dispositif et outils. Dans une perspective vygotskienne, les outils cognitifs ne sont pas seulement matériels, mais aussi langagiers. G. S. Cordeiro et B. Schneuwly (2007) distinguent trois catégories d'outils, outils matériels (tableau, cahiers...), tâches (exercices, activités, problèmes) et « discours élaborés par l'école » sur l'objet enseigné. «Ces outils et leur mise en œuvre en classe, disent-ils, composent le "dispositif didactique" : une série de situations et de supports à des fins didactiques qui instaurent un espace sémiotique au service de l'objet et de ses significations. » (\$ 16) Il s'agit ainsi d'aménager le milieu didactique, « système des objets qui déterminent les pratiques de l'étude des savoirs » (Brousseau, cité par Reuter et al., 2007). Insister sur le rôle du dispositif dans les processus d'enseignementapprentissage, c'est affirmer que les apprentissages, les transformations des modes de 
penser-parler-écrire ne procèdent pas de l'intervention directe de l'enseignant, mais de la médiation que constitue l'organisation et la structuration d'un système d'outils.

On rejoint là le second trait caractéristique du concept, hérité de M. Foucault, la contrainte implicite que fait peser le dispositif sur le public qu'il vise. Des dispositifs éducatifs institutionnels nombreux se sont par exemple succédé depuis une trentaine d'années pour traiter la difficulté scolaire ou lutter contre le décrochage. Les dispositifs, commente A. Barrère (2017), sont "une manière de "faire faire", de contraindre l'action par des stratégies pragmatiques, dépassant le recours à la seule intériorisation des normes et des valeurs " (p. 216). Cette caractéristique des dispositifs a souvent été analysée par les sociologues, tant dans le domaine de l'éducation A. Barrère (2013) parle ainsi d'une "montée des dispositifs" dans ces dernières décennies comme moyens de réformer l'école - que dans d'autres domaines - par exemple lorsque B. Latour (1996) évoque la ceinture de sécurité comme moyen de réduire l'hécatombe routière là où les injonctions règlementaires ont échoué.

Dans le domaine pédagogique, cette importance de l'action indirecte des enseignants sur les élèves par l'organisation d'un milieu renvoie aussi au courant de l'Éducation nouvelle. L'éducation par les choses et par l'expérience que Rousseau entend donner à Émile ne se fait que parce que l'éducateur organise les rencontres de son élève avec les choses et en anticipe les effets. Des mouvements d'éducation aussi différents que les groupes de Pédagogie institutionnelle ou le Groupe français d'éducation nouvelle (GFEN) ont contribué à populariser auprès des enseignants l'idée que changer l'école revient à changer les dispositifs qu'on y met en œuvre, des « institutions » comme le conseil de coopérative permettant, dans le premier cas, de rompre le face-à-face mortifère de l'enseignant et de l'élève (Vasquez \& Oury, 1967), les « démarches » visant, dans le second, à une "autosocioconstruction " des savoirs par les élèves eux-mêmes (GFEN, 1982).

5 Un troisième trait récurrent dans les théorisations des dispositifs éducatifs est son aspect de construction sociale collective, intermédiaire entre les normes édictées par l'institution scolaire et la pratique de terrain (Bonnéry, 2014). Le projet général de S. Bonnéry est de comprendre les difficultés scolaires dont sont sélectivement victimes les enfants des milieux populaires et les formes de domination sociales dont l'école est le théâtre; des «malentendus » (Bautier \& Rochex, 2007), qui font obstacle au travail cognitif des élèves les plus fragiles et les moins acculturés aux attentes de l'école, résultent notamment des dispositifs, de leurs implicites et des interprétations partielles ou erronées qu'en ont ces élèves. S. Bonnéry fait des dispositifs un niveau mésosociologique d'analyse, "intermédiaire entre les influences de la hiérarchie de l'institution scolaire [...] et les influences exercées par les enseignants et les élèves dans les situations locales " (Bonnéry, 2014, p. 4). Si l'on retrouve chez Bonnéry l'idée d'une relation étroite entre les outils - et surtout les supports du travail des élèves, livres, manuels, fiches... - et l'ensemble des tâches et consignes qui les accompagnent, ainsi que celle d'une "régulation à distance " - les modalités d'organisation permettent de faire faire sans intervention directe de l'enseignant - il met surtout l'accent sur le caractère social, et non pas propre à un enseignant, des dispositifs. Ceux-ci « s'imposent à lui [l'enseignant], et constituent [...] l'extension des influences sociales et institutionnelles qu'ils matérialisent" (Bonnéry, 2014, p. 105), qu'il s'agisse de dispositifs sans auteur identifiable (des façons d'enseigner partagées par la profession à 
un moment donné) ou bien d'ingénieries proposées par des groupes ou des personnes, dans une perspective de résolution d'un problème à traiter.

Jusqu'où un dispositif s'impose-t-il aux enseignants qui l'adoptent? C. Viriot-Goeldel (2011), étudiant les dispositifs américains de réponse aux difficultés de lecture des élèves, a montré leur caractère impératif. En revanche, dans le contexte français, les enseignants disposent d'une grande liberté pédagogique pour adapter des dispositifs. Dès lors, il convient de s'intéresser aussi à ces adaptations. La manière de mettre en œuvre un dispositif pourrait être ce qui permet ou non à celui-ci d'atteindre ses buts. C'est la position de C. Delarue-Breton (2012), qui reprend la distinction faite par E. Belin (2002) entre deux aspects des dispositifs: d'une part les conditions matérielles et le séquençage de l'activité, d'autre part les intentions et enjeux - la «logique dispositive ». Or, la visée d'apprentissage est souvent oubliée dans les dispositifs qu'elle analyse. Aussi insiste-t-elle sur la nécessité que la mise en œuvre du dispositif soit pensée, non seulement dans son déroulement, mais en prenant comme fil conducteur du déroulement la logique dispositive.

7 En résumé, mettre en avant le dispositif, tant dans les préconisations que dans les analyses des situations d'apprentissage scolaire, conduit à minimiser le rôle des interventions directes de l'enseignant au profit d'une forme qui modèle à priori la situation, des modes d'organisation de celle-ci et des outils qu'il y met en œuvre. Il est pourtant difficile de faire l'impasse sur le rôle direct des enseignants au cours des interactions didactiques. C'est ce que nous allons tenter d'illustrer dans les analyses qui suivent.

\section{Une recherche sur le dispositif Twictée}

8 Twictée, dispositif numériquement instrumenté d'enseignement et d'apprentissage de l'orthographe, a été créé par des enseignants du primaire et associe aujourd'hui plusieurs centaines de classes francophones d'écoles et de collèges, fédérées en une association. Il repose sur des échanges et des «négociations graphiques » (Brissaud \& Cogis, 2011 ; Haas, 1999) par l'intermédiaire du réseau social Twitter ${ }^{1}$. Appariées par trois, les classes échangent de courtes dictées; les élèves corrigent les dictées de correspondants et reçoivent des corrections élaborées par d'autres correspondants. En classe entière et/ou en petits groupes, ils se mettent d'accord et rédigent l'argumentation justifiant la correction proposée, en s'appuyant sur une liste de catégories d'erreurs. Les dictées et les corrections sont envoyées par tweets, en respectant des contraintes précises, comme nous le verrons plus loin.

9 Nous avons conduit notre étude ${ }^{2}$ auprès de 40 classes d'enseignants volontaires durant l'année scolaire 2017-2018, en régions parisienne et grenobloise. Dans les 19 classes participant au dispositif Twictée, les enseignants et leurs élèves ont pratiqué la Twictée à leur propre rythme durant toute l'année scolaire, sans l'intervention des chercheurs, réalisant entre trois et six Twictées. Dans les 21 classes constituant le groupe témoin, les enseignants ont enseigné l'orthographe avec leurs pratiques habituelles. Des entretiens semi-directifs et des observations de séances ont permis de décrire ces pratiques dans les deux groupes. Les performances des élèves des deux groupes ont été évaluées en début et fin d'année scolaire afin de mesurer les progrès effectués et les progrès des deux groupes comparés. 
10 Les élèves ont passé trois épreuves d'orthographe en septembre 2017 (prétest) et en juin 2018 (posttest).

11 - Une dictée utilisée par la Direction de l'évaluation, de la prospective et de la performance (DEPP) dans son étude diachronique conduite en CM2 (1987-2015) (DEPP, 2016).

12 - Un exercice de transformation, qui consiste à réécrire un texte après avoir mis au pluriel le premier groupe nominal (la sauterelle) et à faire toutes les modifications qui s'ensuivent.

13 - Une production de texte, dont les résultats ne seront pas exploités ici.

14 Un score global a été constitué à partir de l'ensemble des mots correctement orthographiés. Les performances des élèves ont fait l'objet de traitements statistiques (voir Brissaud, Viriot-Goeldel \& Ponton, 2019 pour plus de détails).

\section{Des progrès variables d'une classe à l'autre}

15 L'analyse des progrès des élèves selon qu'ils ont bénéficié ou non du dispositif Twictée, réalisée au moyen d'un modèle statistique multiniveau, permet de vérifier l'hypothèse d'un effet du dispositif toutes choses égales par ailleurs. Nous avons comparé l'évolution du score global d'orthographe entre les élèves des classes twictantes et les élèves des classes témoins. Le tableau ci-dessous expose les résultats de cette évolution entre le début et la fin de l'année scolaire.

\begin{tabular}{|c|c|c|c|c|c|c|c|c|}
\hline \multirow[t]{2}{*}{ Score } & \multicolumn{2}{|c|}{$\begin{array}{l}\text { Groupe } \\
\text { témoin }\end{array}$} & \multicolumn{2}{|c|}{$\begin{array}{l}\text { Groupe } \\
\text { Twictée }\end{array}$} & \multirow{2}{*}{$\mathrm{Chi}^{2}$} & \multirow[t]{2}{*}{ DDL } & \multirow{2}{*}{$\begin{array}{l}\mathrm{p}- \\
\text { value }\end{array}$} & \multirow{2}{*}{$\begin{array}{l}\text { Effect } \\
\text { Size }\end{array}$} \\
\hline & Moy & SD & Moy & SD & & & & \\
\hline $\begin{array}{l}\text { Évolution du score global (T2 - } \\
\mathrm{T} 1)\end{array}$ & 8.20 & 10.97 & 10.49 & 11.58 & 1.311 & 1 & 0.252 & 0.364 \\
\hline
\end{tabular}

Si on note une évolution plus marquée du nombre de mots orthographiés correctement pour le groupe pratiquant la Twictée $(10,49)$ que pour le groupe témoin $(8,20)$, la différence n'est toutefois pas significative.

17 L'absence de significativité des effets moyens constatés invite à se pencher sur les résultats de chaque classe. La répartition des scores de chaque classe, qu'on y pratique ou non la Twictée, montre des différences importantes dans la capacité à faire progresser les élèves. Ainsi, il y a aussi bien des classes twictantes dont les élèves progressent significativement plus que la moyenne (par exemple les classes 16 et $17^{3}$ ) que des classes twictantes dont les élèves progressent significativement moins que la moyenne (par exemple les classes 12 et 23).

18 Nous nous tournerons donc vers d'autres données pour tenter de comprendre ces variations : ce que disent les enseignants lors des entretiens, les différences dans la mise en œuvre du dispositif et la qualité des interventions des enseignants au cours des séances de travail. 


\section{Ce que disent les enseignants : formalisme et motivation}

$19 \mathrm{Au}$ cours d'entretiens semi-directifs d'une heure, les enseignants étaient invités à décrire leurs pratiques, leurs manières d'enseigner et les transformations éventuelles de celles-ci, leurs conceptions de la langue et de l'enseignement, leur rapport à la discipline, leurs perceptions des compétences des élèves, leurs modes de collaboration avec les collègues. Le contenu de ces entretiens a été analysé en catégories thématiques. De cette analyse, présentée ailleurs (Cadet, Crinon \& Ferone, 2019), nous reprendrons ici des éléments relatifs au dispositif.

\section{Négocier}

20 De fait, dans les entretiens, le recours à la négociation, l'argumentation ou la justification construite au cours de discussions est très souvent mis en avant par les enseignants participants. Ils en attendent surtout un entrainement au " raisonnement orthographique » que doivent appliquer les élèves lorsqu'ils écrivent et lorsqu'ils se relisent.

l'objectif il va être toujours de réfléchir à ce qu'on écrit d'un point de vue donc du coup à les confronter à ce qu'ils argumentent à ce qu'on discute [...] l'élève qui vient au tableau peu importe s'il a dix erreurs il doit être capable d'argumenter et souvent quand il est au tableau il arrive à se corriger de lui-même quand il a ce processus de réflexion autour des mots il va réussir à se corriger $\left(\mathrm{C} 16^{4}\right)$

21 Habitudes de travail, automatisation de ce raisonnement, listes de questions à se poser, sont donc des expressions qui reviennent fréquemment dans leurs propos. Il en résulte une sorte d'algorithme, modélisé par l'enseignant et reproduit ensuite par les élèves.

qu'ils aient des automatismes en fait quand ils font des dictées regarder les noms le pluriel l'adjectif chercher le verbe par exemple on travaille beaucoup sur quand on a un texte on cherche le verbe d'accord une fois qu'on a le verbe qu'est-ce qu'on fait on cherche les méthodes (C21)

qu'ils aient justement ce réflexe c'est qu'ils aient un réflexe orthographique qu'ils aient une espèce de gymnastique qui puisse après leur permettre de se vérifier tout seul (C22)

Ces procédures à appliquer seront d'autant plus facilement mémorisées et automatisées qu'elles seront répétées. Elles sont donc systématiquement sollicitées, aussi bien lors des séquences Twictée qu'à toutes les autres occasions, qu'il s'agisse d'exercices d'orthographe ou de productions d'écrits. En outre lors d'une séquence Twictée, la correction justifiée de la même dictée est elle-même répétée à plusieurs reprises, notamment lors d'une première correction collective et lors de l'écriture des corrections argumentées envoyées aux correspondants (les «twoutils» dans la terminologie du dispositif). Cette conception du rôle de la négociation est cohérente avec la récurrence du thème de la mémorisation par la répétition, l'entrainement, voire le « rabâchage », thème le plus fréquent dans le corpus d'entretiens.

La forme adoptée, contraignante et récurrente, a, elle aussi, son importance. S'adresser à des partenaires distants amène à expliciter la correction par écrit, en outre toujours selon le modèle donné sur le site de l'association : 


\title{
Écrire un bon \#Twoutil
}

\section{@classescribe \#Twoutil «mot correctement orthographié» s'écrit " correction » car [Justification]. \#balise}

\author{
Ex " enfants " s'écrit avec un -S car il y a «les" \\ déterminant pluriel placé devant. \#AccordGN
}

Plusieurs des personnes interviewées insistent sur cet aspect.

ce qui est intéressant c'est l'échange avec les autres essayer d'être le plus clair dans mes justifications pour que ça puisse être utile (C17)

la twictée prend du temps les quatre étapes elles sont longues et surtout le la création des twoutils où c'est le cœur du dispositif (C16)

quand tu crées un twoutil il faut quand même que ce soit très précis euh il y a vraiment la phase justification catégorisation de l'erreur et avec utiliser tous les termes [...] alors que quand tu fais la négociation euh à l'oral quand tu fais la phrase du jour et la négociation à l'oral il me semble que cette précision elle n'y est pas forcément toujours (C43)

L'accent mis sur l'apprentissage de procédures stabilisées permettant de vérifier et corriger l'orthographe de ses productions va de pair avec ce que disent les enseignants des points forts et des points faibles de leurs élèves. Ce qui met en échec les élèves, selon eux, ce n'est pas l'absence des connaissances nécessaires, mais la difficulté à mobiliser celles-ci en situation.

ils vont très bien enregistrer les homophones grammaticaux a ou à ils vont très bien maitriser dans un exercice où il faut compléter par contre dès qu'on va passer sur une dictée ou sur de l'écriture spontanée là ils ont vraiment du mal à réinvestir (c22)

Cela peut aussi expliquer qu'un tout petit nombre d'enseignants seulement évoque la possibilité d'accéder aux représentations orthographiques des élèves et de les faire évoluer grâce aux négociations. Ils n'envisagent pas que des représentations inadaptées ou partielles et une absence de clarté sur le fonctionnement du système puissent faire obstacle à une écriture conforme à la norme. Pour les enseignants qui le pratiquent, la première vertu du dispositif semble de contraindre à un schéma de raisonnement explicite.

\section{Motiver}

Un autre thème récurrent dans les entretiens est la recherche de la motivation. Ce désir de motiver les élèves, voire de les réconcilier avec une discipline traumatisante est une 
des raisons invoquées lorsqu'on demande ce qui les a poussés à s'engager dans la Twictée.

mes attentes c'était premièrement de motiver les élèves sur cette question de l'orthographe qui était absolument pas motivante maintenant ils sont contents de faire de l'orthographe (C44)

\section{des leviers.}

contrairement à moi petit je pense qu'ils voient plus le côté ludique dans l'exercice autour de la twictée c'est vraiment un jeu quoi on va corriger d'autres enfants qui sont loin de nous on travaille pas pour le maitre (C16)

31 Très fréquemment aussi les enseignants évoquent les affects négatifs liés à l'orthographe chez les élèves, parfois en faisant référence à leur propre passé d'élève : à l'orthographe sont associés des termes forts comme souffrance, stress, traumatisme, tragique... Il faut donc rassurer, dédramatiser.

moi petit j'ai eu des moins quarante en dictée traumatisant là ils ont juste voilà j'ai fait six erreurs j'ai fait dix erreurs (C16)

[les élèves en difficulté,] il leur manque plein de choses en fait c'est pas que dans l'orthographe c'est aussi dans leur manière d'être élève etc. donc il faut les rassurer beaucoup (C21)

comme on a beaucoup réfléchi collectivement pour corriger je vous dis pour bien prendre conscience des réflexions des questions qu'on doit se poser quand on fait une dictée en général c'est bien réussi donc c'est plutôt gratifiant pour eux (C22)

$\mathrm{Au}$ total, les enseignants expriment leur confiance dans le pouvoir de ce dispositif à faire progresser les élèves, grâce aux contraintes qui lui sont inhérentes (une justification systématique des corrections d'erreurs de manière très formalisée et codifiée), à la répétition des mêmes tâches et à l'adhésion qu'il emporterait chez les élèves. En revanche, ils évoquent peu la manière dont ils sont eux-mêmes présents dans les interactions d'apprentissage, si ce n'est par l'installation du cadre et des supports et la verbalisation des modèles de raisonnement.

\section{Les mises en œuvre : variation autour d'une trame}

Le dispositif de la Twictée comporte six phases principales que l'on peut caractériser de la façon suivante :

1. une courte dictée individuelle ;

2. une négociation entre pairs permettant d'adresser à une classe partenaire des productions de groupes;

3. une correction collective du texte ;

4. la correction des textes de partenaires avec rédaction de twoutils ;

5. la correction des productions de groupe à partir des twoutils reçus ;

Pratiques, $189-190 \mid 2021$ 
6. une dictée dite de transfert (facultative).

34 Mais l'analyse du travail effectivement conduit dans quatre classes $(12,16,17,23)$ laisse apparaitre des mises en œuvre assez différentes. Le tableau ci-dessous en fait apparaitre les principales variations entre les classes 12 et 17 à titre d'exemple ${ }^{5}$.

\begin{tabular}{|c|c|c|}
\hline $\begin{array}{l}\text { Phases de } \\
\text { la twictée }\end{array}$ & Séquence de la classe 17 & Séquence de la classe 12 \\
\hline 1 & $\begin{array}{l}\text { Consignes et dictée individuelle (13 } \\
\text { minutes) }\end{array}$ & Consignes et dictée individuelle (5 mn 15) \\
\hline 2 & Négociation en petits groupes ( $32 \mathrm{mn}$ ) & Négociation en petits groupes (14 mn 35) \\
\hline 3 & Correction en groupe classe ( $55 \mathrm{mn})$ & Correction en groupe classe (17 mn 50) \\
\hline \multirow{2}{*}{4} & $\begin{array}{l}\text { Repérages des erreurs de la classe } \\
\text { partenaire en petits groupes et mise } \\
\text { en commun }(44 \mathrm{mn})\end{array}$ & $\begin{array}{l}\text { Repérage des erreurs de tous les groupes de la } \\
\text { classe partenaire (travail individuel) et mise } \\
\text { en commun collective ( } 34 \mathrm{mn} \text { ) }\end{array}$ \\
\hline & $\begin{array}{l}\text { Rédaction des twoutils en petits } \\
\text { groupes et envoi à une classe } \\
\text { partenaire }(36 \mathrm{mn})\end{array}$ & $\begin{array}{l}\text { Consignes, exemple et rédaction de deux } \\
\text { twoutils par binômes }(38 \mathrm{mn})\end{array}$ \\
\hline \multirow{3}{*}{5} & $\begin{array}{l}\text { Correction collective d'une twictée } \\
\text { avec recherche des balises ( } 10 \mathrm{mn})\end{array}$ & - \\
\hline & $\begin{array}{l}\text { Lecture et explication des twoutils } \\
\text { reçus ( } 13 \mathrm{mn})\end{array}$ & - \\
\hline & $\begin{array}{l}\text { Correction des dictées de groupe à } \\
\text { l'aide des twoutils reçus ( } 30 \mathrm{mn})\end{array}$ & - \\
\hline 6 & $\begin{array}{l}\text { Twictée de transfert et autocorrection } \\
\text { (9 mn) }\end{array}$ & - \\
\hline $\begin{array}{l}\text { Temps } \\
\text { total }\end{array}$ & 242 minutes & 109 minutes \\
\hline
\end{tabular}

Les deux enseignants regroupent les trois premières phases de la Twictée en une séance. La séance se déroule de façon similaire, et conformément au schéma prévu par les concepteurs du dispositif. Elle débute par une dictée individuelle, suivie d'une négociation orthographique en petits groupes puis d'une correction en classe complète.

Plusieurs différences apparaissent ensuite. L'enseignant de la classe 17 ajoute par exemple deux phases de son invention: un exercice de correction collective d'une twictée avec recherche des balises rendant compte des erreurs, ainsi qu'un temps de lecture et d'explication des twoutils reçus. De son côté, l'enseignant de la classe 12 fait recopier aux élèves la twictée corrigée sur le cahier à la fin de la première séance. Cela modifie ainsi sensiblement le travail cognitif de repérage des erreurs prévu par les créateurs de la Twictée. L'enseignant de la classe 12 ne fait pas corriger aux élèves leur propre production à partir des twoutils reçus, cas assez inhabituel dans notre échantillon. Comme près de la moitié des classes observées, il ne réalise pas non plus la twictée de transfert. Ces deux exemples de déroulement témoignent bien de la marge de manœuvre des enseignants, qui aménagent le dispositif à leur manière. 

du maitre qui peut consacrer le temps qu'il souhaite aux différentes composantes de la Twictée. Alors que, dans la classe 17, 100 minutes sont consacrées à la dictée, la négociation et la correction, les élèves de la classe 12 réalisent ce travail en seulement 37 minutes. On conçoit dès lors combien le nombre d'interactions consacrées à la construction des compétences orthographiques diffère entre les deux classes. L'examen de deux autres classes (16 et 23) (Crinon \& Viriot-Goeldel, 2019) laisse apparaitre des variations du même type. Dans la classe 16, ce sont 94 minutes qui sont consacrées au travail collaboratif entre pairs, soit presque la moitié de la durée de la séquence. Le maitre est loin d'être un simple exécutant comme dans certains dispositifs "clés en main » très minutés dans lesquels il conduit une série d'exercices imposés ${ }^{6}$ (ViriotGoeldel, 2011).

ositif, qui paraissait contraignant, ne constitue finalement qu'une trame que les enseignants adaptent assez librement, créant des séquences à l'intérieur desquelles des durées très importantes sont en outre consacrées aux interactions en groupe classe et entre pairs. Il semble largement reposer sur la capacité des enseignants à conduire des interactions autour des problèmes orthographiques, et ces interactions, nous allons le voir maintenant, se révèlent aussi très différentes d'une classe à l'autre. On s'attend dès lors à ce que la variation de l'efficacité du dispositif d'un maitre à l'autre dépende aussi de ces interactions langagières.

\section{Dans la classe : argumenter l'orthographe}

\section{L'installation de procédures}

Les enseignants mettent souvent l'accent sur l'enseignement de procédures, séries d'étapes à suivre pour raisonner sur la graphie à transcrire. C'est le cas dans la classe 16, un CM1 (voir Crinon \& Viriot-Goeldel, 2019). Dans la classe 17, un CM2 de la même école, ces deux procédures sont déjà bien installées, les élèves les mobilisent spontanément en situation de Twictée sans que l'enseignant ait besoin de les rappeler. D'ailleurs, à plusieurs reprises, l'enseignant félicite les élèves de leur maitrise de l'accord au sein du groupe nominal (« accord GN, on a l'impression que ça commence à bien rentrer », "oui, vous avez bien pensé à l'accord GN »), témoignant ainsi de sa satisfaction devant la mobilisation systématique de ces procédures.

À l'instar de l'enseignant de la classe 16, l'enseignant de la classe 17 fait chercher systématiquement la nature des mots à écrire, procédure qui permet ensuite de déterminer la règle d'accord à utiliser, comme dans l'exemple qui suit. Dans la phase 5 de la Twictée, il mobilise cette procédure à quatre reprises.

les seigneurs noirs

$\mathrm{M}^{7}$ : noirs s'écrit sans $\mathrm{E}+$ pourquoi?

$\mathrm{E}$ : parce que euh c'est les seigneurs noirs et on dit un seigneur

$M$ : oui essaye de me dire sa nature à ce mot

$\mathrm{E}:$ c'est un adjectif

$\mathrm{M}:$ donc il va s'accorder avec euh le nom qui est au +

$\mathrm{E}:$ masculin pluriel

$\mathrm{M}:$ masculin pluriel + formidable [...]

$\mathrm{E}$ : les seigneurs noirs combattent

$\mathrm{M}$ : essaie de m'expliquer pourquoi c'est ENT et pas ES

$\mathrm{E}:$ parce que son sujet est au pluriel

Pratiques, $189-190 \mid 2021$ 
$\mathrm{M}$ : alors son sujet ça veut dire que lui c'est quoi ce mot ? c'est quoi sa nature ?

$\mathrm{E}$ : combattent c'est un verbe

$\mathrm{M}$ : voilà + combattent c'est un verbe + son sujet ?

$\mathrm{E}:$ il est au pluriel

$\mathrm{M}:$ il est au pluriel

E : ça s'accorde au pluriel

$\mathrm{M}$ : donc ça va être quoi ma balise ? tu m'as parlé de verbe, tu m'as parlé de sujet +

$\mathrm{E}$ : hashtag accord sujet verbe (C17)

Une autre procédure encouragée plusieurs fois par l'enseignant de la classe 17, lors de la phase 3, consiste, quand on rencontre des difficultés à écrire un mot, à déterminer s'il s'agit d'un problème d'orthographe grammaticale ou lexicale, afin, dans un deuxième temps, d'opter pour un raisonnement à l'échelle de la phrase ou une recherche dans le dictionnaire.

C'est ainsi le cas lorsqu'il s'agit de trouver la terminaison de engagées :

$\mathrm{M}$ : alors, est-ce que là on a besoin du dictionnaire ?

$\mathrm{E}:$ non

$\mathrm{M}$ : là c'est un problème de quoi ? est-ce qu'on a un problème sur l'orthographe lexicale?

E : non

$\mathrm{M}$ : donc on n'est pas du tout dans un problème d'orthographe lexicale, donc le dictionnaire il va nous être d'aucune aide là donc là + on est sur un problème de terminaison c'est de l'orthographe grammaticale il va falloir que je regarde autour de ma phrase (C17)

C'est encore le cas lorsqu'il s'agit de s'interroger sur les deux T de combattre:

$\mathrm{M}$ : pourquoi c'est un problème qui va nous poser problème à nous justifier ça ? c'est un problème de quel type d'orthographe?

E : lexicale

$\mathrm{M}$ : lexicale et à chaque fois cette orthographe-là elle nous pose problème + pourquoi ? pourquoi elle pose problème celle-ci ? pourquoi elle est plus compliquée à justifier qu'un autre un problème d'orthographe grammaticale Leïla?

$\mathrm{E}$ : un problème d'orthographe grammaticale on doit pouvoir montrer pourquoi ça s'écrit comme ça alors que le champ lexical on peut pas

$\mathrm{M}$ : et comment je montre pourquoi dans un problème d'orthographe grammaticale?

$\mathrm{E}$ : on cherche le sujet

$\mathrm{M}$ : oui on cherche dans la

E : phrase

$M$ : est-ce que là ça va m'aider de chercher dans la phrase

$\mathrm{E}:$ non

M : non hein je vais être obligé de réfléchir juste sur le mot [...] j'ai pas la justification sous les yeux vas-y Tracy

E : (inaudible)

$\mathrm{M}$ : d'accord tu dis qu'on ne double pas forcément la consonne entre deux voyelles c'est ça que tu dis, d'accord Kylian?

$\mathrm{E}$ : c'est avec un $\mathrm{T}$ parce que ça vient du mot combat + c'est un mot où on rajoute une syllabe

$\mathrm{M}$ : oui tiens d'ailleurs on a rajouté quoi devant? comment ça s'appelle ça ? un préfixe + on a rajouté le préfixe à quel verbe ? au verbe battre + donc vous étiez nombreux à me dire que ça vient du verbe battre du coup on va chercher quelle orthographe? l'orthographe du verbe battre alors on va faire confiance à Kayla qui a eu la bonne idée de sortir son dictionnaire + c'est un problème d'orthographe lexicale on n'arrive pas à se mettre d'accord on regarde dans le dictionnaire (C17) 


\section{Des procédures que l'on cherche à automatiser}

automatismes.

$\mathrm{M}$ : ils ont été précis ils ont été chercher la nature ensuite ils ont dit que ça précisait le mot vies ensuite ils ont dit que vies c'était du féminin pluriel allez c'est réglé c'est tellement plus simple [...] à condition d'aller chercher la nature tout de suite après le mot + et après ça se déroule tout seul un petit peu comme un livre + voilà j'ai la nature je tourne les pages après il me faut le nom il me faut la nature et le genre ça marche ? [...] oui il manque la nature et comme vous avez pas écrit la nature vous êtes partis sur des chemins + vous auriez trouvé la nature c'était parti vous me le faisiez en deux minutes le twoutil + ça c'est une habitude qu'il va falloir prendre + alors $\mathrm{OK}$ c'est la deuxième twictée alors troisième et quatrième il va falloir après le car maintenant je veux la nature + parce que quand vous avez la nature ensuite vous savez ce que vous avez à faire (C16)

Pour automatiser le recours à des schémas de raisonnements, les élèves sont ainsi invités à les répéter à l'identique au cours des séances successives de la Twictée. Le caractère ritualisé du dispositif facilite la mise en place de ces procédures, par la répétition des mêmes formats de tâches, des mêmes supports, des mêmes consignes.

La répétition est de mise également dans la séance de construction des twoutils, au cours de laquelle les interventions du maitre poussent à faire retrouver ce qui a été dit dans la séance collective précédente.

- rappelez-vous pourquoi on avait dit qu'il fallait deux $G$

- nous on avait expliqué pourquoi c'était une erreur après c'est à vous de vous

rappeler ce qu'on avait dit + au moment de la correction

- on avait expliqué Max la semaine dernière

- il faut se rappeler de ce qu'on a dit la semaine dernière

- ça dort ce matin tout est au tableau y a juste à regarder au tableau (C16)

4 L'étayage du maitre consiste ainsi à faire rappeler les solutions trouvées ensemble ou données par lui-même dans des séances précédentes. Il s'agit donc bien de faire appel à la mémoire : mémoire des solutions, mémoire de la procédure à mettre en œuvre pour arriver à la solution. Acquérir l'orthographe, pour cet enseignant (comme pour beaucoup d'autres dans la population observée) repose essentiellement sur la mémorisation, dont la répétition est gage. La séance de rédaction de twoutils est donc un moment de répétition, où il s'agit en premier lieu de retrouver les solutions et les justifications déjà trouvées et données précédemment. Les interventions de l'enseignant lorsqu'il fait le tour des groupes vont dans ce sens.

\section{Des interventions de qualité inégale}

Mais, au-delà de ces points communs, on trouve aussi des différences notables dans la façon dont les enseignants tentent d'installer et d'automatiser ces procédures. Si l'on considère l'exemple de la manipulation qui consiste à remplacer une forme verbale en / $\mathrm{E} /$ par un verbe du deuxième ou troisième groupe afin de déterminer s'il s'agit d'un infinitif ou d'un participe passé, les explications des enseignants se font parfois confuses. L'examen des séances de la classe 16 avait montré la difficulté pour l'enseignant de conduire la discussion collective afin de décider si [partaze] est un infinitif ou un participe passé (Crinon \& Viriot-Goeldel, 2019). Dans la classe 17, les procédures de manipulations semblent mieux maitrisées. Alors que dans certains 
autres exemples, cette manipulation s'apparente plus à une astuce à mettre en œuvre sans en comprendre les enjeux, le recours spontané à ces procédures par les élèves de la classe 17, en utilisant à bon escient le métalangage grammatical, montre qu'elles sont installées, comme dans cet exemple où deux élèves utilisent les termes verbe, adjectif, préposition et infinitif pour justifier la terminaison ER de dominer :

$\mathrm{E}:$ on peut remplacer par un verbe c'est pas un adjectif de galaxie si on dit pour dominer + dominer c'est un verbe on peut remplacer par le verbe tondre ou prendre et quand on dit prendre tondre c'est $\mathrm{E} R$ à la fin

$\mathrm{M}:$ qui a autre chose à rajouter?

$\mathrm{E}:$ moi je pense que c'est dominer $\mathrm{E} R$ parce que pour c'est une préposition et comme un verbe après une préposition il est à l'infinitif je pense que c'est $\mathrm{E} R$ (C17)

Dans plusieurs classes, en revanche, apparaissent des erreurs de raisonnement grammatical commises ou validées par le maitre : ainsi, dans la classe 12, une confusion entre les notions de « mot invariable » et d'« orthographe d'usage».

voilà

M : alors pourquoi on écrit alors pourquoi c'est une faute pourquoi enfin c'est une faute pourquoi c'est une erreur pourquoi il faut effectivement un accent qui est-ce qui le sait qui est-ce qui peut nous apprendre quelque chose ? oui

$\mathrm{E}$ : parce que c'est un mot invariable

$\mathrm{M}$ : parce que c'est un mot invariable et que ça s'écrit comme ça (classe 12)

Un autre exemple serait la confusion entre les notions d'« orthographe d'usage » et « accord GN » pour justifier le E final de galaxie.

$\mathrm{M}$ : vous avez entendu + oui alors elle pense que galaxie c'est au féminin donc on met un $\mathrm{E}$ à la fin vas-y Almet

$\mathrm{E}:$ ici il y a pas de $\mathrm{E}$

$\mathrm{M}$ : l'absence de $\mathrm{E}$ ça correspond à quoi comme balise on va mettre les balises au fur et à mesure

$\mathrm{E}$ : lettre son

$\mathrm{M}$ : ah bon ça change quelque chose à la façon de le prononcer ou pas

E : non

E : accord GN

E : non

$\mathrm{E}:$ lettre manquante

$\mathrm{M}$ : lettre manquante et comment on peut le justifier ça c'est grâce au quoi

$\mathrm{E}:$ au déterminant

$\mathrm{M}$ : donc c'est quoi

$\mathrm{E}$ : accord GN

$\mathrm{M}$ : est-ce que c'est juste une lettre qui manque ou elle a un sens cette lettre?

$\mathrm{E}$ : elle a un sens

$\mathrm{M}$ : est-ce qu'elle a un sens grammatical

$\mathrm{E}$ : oui

E : non

$\mathrm{M}$ : bah oui elle est là pourquoi parce qu'on est au féminin donc qu'est-ce qu'on va pouvoir utiliser?

$\mathrm{E}$ : hashtag accord GN

$\mathrm{M}$ : alors on va pouvoir utiliser éventuellement hashtag accord GN et pourquoi pas coupler avec un autre hashtag donc c'est un problème de lettre manquante donc en vert on va avoir un problème d'accord (Classe 17). 


\section{Le travail sur les justifications erronées}

51 Lors des échanges collectifs, les élèves émettent souvent des propositions erronées. Ainsi, "galaxie avec une majuscule, c'est faux, parce que galaxie, c'est pas un mot important " et «c'est pas un grand mot »; "Le mot combat il y a un seul $T$ donc on rajoute peut-être juste re "; pour combattre, "battre avec deux $T$ parce que c'est un verbe avec conjugaison"; auprès, "c'est en un seul mot parce que si tu enlèves le U ça fait après "; Guerre (dans Guerre des étoiles), c'est un nom propre parce que "c'est une guerre importante ", Guerre s'écrit avec une majuscule "car c'est un grand évènement » : ces justifications erronées font en général l'objet de discussions où l'enseignant essaie d'amener l'élève à expliciter son raisonnement, puis apporte la graphie correcte, souvent en sollicitant d'autres élèves.

$\mathrm{E}$ : moi je pense pas que c'est Galaxie avec une majuscule parce que c'est pas un grand mot

$\mathrm{M}$ : c'est pas un grand mot parce que il y a pas beaucoup de lettres ou c'est quoi un grand mot

E : un nom propre

$\mathrm{M}:$ nom propre d'accord tu penses que c'est pas un nom propre Nolan [...]

E: Galaxie avec une majuscule c'est faux parce que galaxie c'est pas un mot important

$\mathrm{M}$ : qu'est-ce que tu entends par mot important là on a entendu grand mot mot important + comment tu les appelles ces mots-là + elle pense que c'est quoi en fait Morgane elle a pas le bon mot mais elle pense que c'est un

$\mathrm{E}:$ nom commun

$\mathrm{M}$ : nom commun c'est là hein que se passe le débat est-ce que c'est un nom propre est ce que c'est un nom commun vas-y

$\mathrm{E}: \mathrm{XXX}$

$\mathrm{M}: \mathrm{bah}$ tu as un avis très complet sur la chose merci Tracy tu en penses quoi toi Malik

$\mathrm{E}: \mathrm{XXX}$

$\mathrm{M}$ : ah c'est bien tu as fait un résumé de la pensée de de Tracy + on se met d'accord

$\mathrm{E}$ : oui galaxie $\mathrm{E}$

$\mathrm{E}: \mathrm{IE}$

$\mathrm{M}$ : alors qui pense qu'il faut une majuscule on va commencer par ça qui pense qu'il ne faut pas de majuscule d'accord là je suis d'accord avec vous on parle de la galaxie

la nôtre on ne met pas de majuscule c'est bien un nom commun (Classe 17).

52 L'enseignante fait expliciter le terme grand mot, auquel elle substitue celui de nom propre sans explication. Elle établit dans un deuxième temps que galaxie est un nom commun.

D'autres justifications erronées sont en revanche soit ignorées, soit même commentées d'une façon qui peut être comprise comme une validation.

combattre

$\mathrm{E}:$ Le mot combat il y a un seul $\mathrm{T}$ donc on rajoute peut-être juste re

$\mathrm{M}$ : D'accord Yanis (Classe 17)

54 Ce dispositif, fondé sur la confrontation des solutions proposées par les élèves, repose ainsi largement sur l'expertise de l'enseignant à choisir, au sein d'une profusion de raisonnements erronés, ceux qu'il traite, puis à apporter un étayage pertinent.

\section{Construire les twoutils entre pairs}

Il convient aussi de s'interroger sur le sort de ces justifications erronées, qui parfois conduisent à la bonne orthographe, lorsque l'enseignant n'est pas présent pour guider 
le raisonnement, lors des négociations en petits groupes. Une des spécificités de la Twictée est la construction des twoutils. Ces derniers comportent une phrase de justification de l'orthographe du mot et une balise ${ }^{8}$. Le choix de la balise requiert en principe l'identification du problème orthographique, et cette identification jouerait un rôle central dans l'acquisition du raisonnement orthographique. Or cette identification est souvent contournée.

Ainsi la balise est parfois choisie par simple association de mots.

$\mathrm{E} 1$ : se s'écrit $\mathrm{S} E$ car derrière il y a un verbe

$\mathrm{M}$ : il accompagne un verbe + très bien + hashtag ?

$\mathrm{E} 1$ : accord sujet verbe

$\mathrm{M}:$ non + où est-ce que tu as entendu le sujet dans sa phrase ? là j'hésite entre ce et

se + quand on hésite entre deux mots c'est?

E2: hashtag homophones (Classe 16)

57 Dans son analyse des corpus d'échanges au sein des petits groupes dans des classes pratiquant la Twictée, P. Fenoglio (2019) note ce qu'elle nomme des phénomènes de "piochage ": les élèves choisissent une balise au hasard dans la liste mise à leur disposition, puis une autre si le groupe pense qu'elle ne convient pas, jusqu'à ce qu'un accord soit trouvé. L'échange ci-dessous s'y apparente : le savoir orthographique n'est pas évoqué et des balises sont piochées successivement dans le document de référence, le maitre encourageant même cette façon de faire.

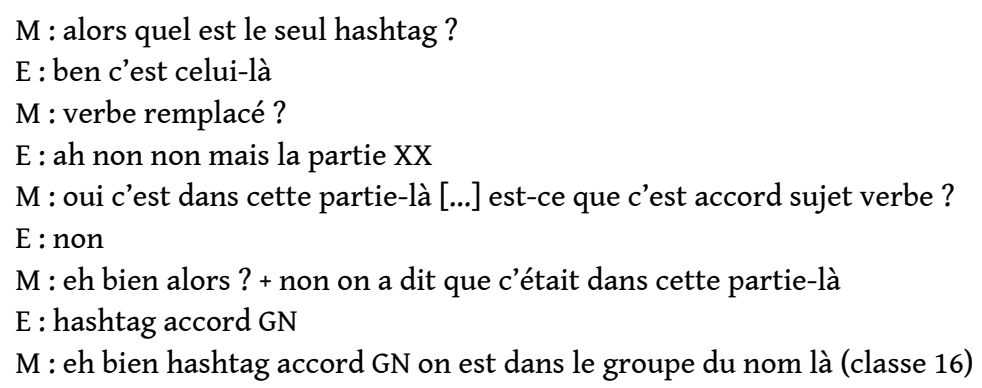

Autrement dit, le dispositif propose des tâches telles que choisir une balise, qui supposent une activité intellectuelle spécifique: identifier le phénomène orthographique. Mais il arrive que les tâches soient effectuées par les élèves sans que l'activité intellectuelle qui y est associée soit mise en œuvre.

\section{Conclusion}

59 À l'issue de cette analyse, il ne s'agirait pas de prétendre qu'adopter tel ou tel dispositif pour atteindre des objectifs pédagogiques et didactiques n'aurait pas d'importance. Les outils, les médiations, les consignes, la structuration de la situation dans l'espace et dans le temps ouvrent des possibles et ont des conséquences sur les apprentissages des élèves. Mais il convient aussi de s'intéresser à ce que les enseignants qui mettent en œuvre le dispositif en attendent et en comprennent et au déroulement réel de la classe, pour rendre compte des manières dont les élèves s'en emparent et y trouvent des occasions pour s'approprier des connaissances et des modes de réflexion. Est particulièrement importante l'intervention de l'enseignant lui-même : les savoirs qu'il apporte et le moment où il les apporte, ses modes de régulation des échanges entre élèves, sa capacité à recentrer l'attention sur les objets pertinents et à synthétiser les 
avancées du travail, ses cadrages, ses étayages, son aide à l'appropriation des outils, sa prise en compte de ce que les élèves comprennent et font du dispositif.

Les enseignants de notre échantillon pratiquant la Twictée mettent deux éléments en avant dans leur discours : d'une part, l'automatisation du recours à des schémas de raisonnement orthographique grâce à la formulation de justifications et à un formalisme imposé dans les tweets envoyés aux partenaires; d'autre part, l'intérêt et la motivation des élèves. En revanche, ils évoquent très peu la manière de faire construire ou évoluer les savoirs orthographiques et peu les étayages apportés, notamment dans le travail de groupes.

61 Ces attentes assez homogènes par rapport au dispositif n'empêchent pas de nombreuses variations d'un enseignant à l'autre, tant dans l'organisation de la séquence de Twictée que dans la conduite de ses différentes phases, qu'il s'agisse de la clarté des arguments évoqués et/ou retenus et de leur justesse, des modalités de reformulation et de répétition du maitre, ou encore des modalités de validation des propositions des élèves. On fera l'hypothèse que ces modalités d'intervention de l'enseignant ont eu une influence importante sur l'activité propre des élèves et, ce qui reste à explorer, sur les progrès que nous avons mesurés chez ceux-ci entre le début et la fin de l'année, progrès qui varient d'une classe à l'autre, tant dans les classes pratiquant la Twictée que dans les autres classes observées.

62 Finalement, Twictée est un dispositif très structuré, mais une large place y est faite aux interactions, notamment lors des séances de correction de la dictée et de construction des twoutils. C'est dans ces interactions que semble se faire la différence. L'appui sur le concept de dispositif dans les recherches en didactique et plus généralement dans les études consacrées aux situations scolaires d'enseignement-apprentissage a le mérite de mettre en évidence l'importance de la structuration de la situation et du choix d'outils et de supports aux potentialités cohérentes avec les objectifs poursuivis. Mais il serait illusoire de croire que la situation et la mise en activité des élèves suffiraient pour que les élèves apprennent, l'enseignant étant supposé se mettre en retrait.

Se pose alors la question de ce qui différencie un dispositif tel que la Twictée, laissant une large marge de manœuvre aux enseignants, d'un dispositif plus contraignant, tel que, par exemple, le programme américain Reading Recovery, dispositif minuté dont chaque enseignant reproduit très exactement le déroulement prévu (Viriot-Goeldel, 2011).

64 Les enseignants du primaire utilisent de nombreux ouvrages qui proposent des dispositifs. Présentant « une série de situations et de supports à des fins didactiques qui instaurent un espace sémiotique au service de l'objet et de ses significations " (Brousseau, cité par Reuter et al., 2007), ils offrent des séances clé en main extrêmement détaillées, alternant outils, supports, consignes, tâches à donner aux élèves et propositions d'organisation du travail. Ils traitent par exemple de la phonologie (Phono: Goigoux, Cèbe \& Paour, 2004 ; ou Phonoludos : Lambert \& Doyen, 2005), ou encore de la compréhension (Narramus MS-GS: Cèbe \& Goigoux, 2017; ou Compréhension, Grande section: Bianco, Coda \& Gourgue, 2002). Peut-on, à l'instar de Twictée et Reading Recovery, les classer en deux catégories, selon qu'ils laissent ou non aux enseignants une certaine liberté dans l'appropriation et la mise en œuvre?

Une enquête sur la transposition de Narramus dirigée par Goigoux (2020) conclut que les propositions didactiques et pédagogiques introduites par Narramus sont reprises par 
les enseignantes lors de la lecture d'autres albums. Ces transpositions font l'objet d'aménagements: les enseignantes reprennent certaines des techniques qui leur étaient proposées mais en abandonnent d'autres et cela, disent les auteurs, contribue à leur développement professionnel. Dans d'autres cas en revanche, ces modifications ne sont pas souhaitées, comme pour l'utilisation de Phonoludos ou de Compréhension Grande Section dans une expérimentation (Bianco et al., 2010) ou de leur mise en œuvre à plus grande échelle dans le cadre du protocole Agir pour l'école (Écalle et al., 2019).

Il s'agit en fait moins de distinguer des catégories de dispositifs que des conditions d'appropriation et de mise en œuvre de ceux-ci. On pourrait alors parler de mise en œuvre créative des dispositifs lorsque ceux-ci peuvent être transformés par les acteurs, et de mise en œuvre contrainte des dispositifs lorsque des règles extérieures empêchent les modifications des contenus et des déroulements.

Quel type de mise en œuvre privilégier ? C'est une question clivante dans le champ de la recherche en éducation, entre ceux qui, pour améliorer l'enseignement, préfèrent réduire la marge de manœuvre de l'enseignant, et ceux qui misent sur son intelligence des situations.

\section{BIBLIOGRAPHIE}

BARRÈRE, A. (2013). «La montée des dispositifs : un nouvel âge de l'organisation scolaire ». Carrefours de l'éducation 36 (2), p. 95-116. En ligne : https://doi.org/10.3917/cdle.036.0095. BARRÈre, A. (2017). « Dispositifs éducatifs ». In van Zanten A. \& Rayou P. (dirs), Dictionnaire de l'éducation, $2^{e}$ édition. Paris : Presses universitaire de France, p. 216-218.

BAUTIER, E. \& ROCHEX, J.-Y. (2007) [1997]. « Apprendre : des malentendus qui font les différences ». In Deauvieau, J. \& Terrail, J.-P. (dirs), Les sociologues, l'école et la transmission des savoirs. Paris : Éditions La Dispute, p. 227-241.

BELIN,E. (2002). Une sociologie des espaces potentiels. Logique dispositive et expérience ordinaire. Bruxelles : De Boeck.

BIANCO, M., CODA, M. \& GOURGUE, D. (2002). Compréhension, Grande section. Grenoble : Éditions de la Cigale.

BIANCO, M., BRESSOUX, P., DOYEN, A.-L., LAMBERT, É., LIMA, L., PELLENQ, C. \& ZORMAN M. (2010). « Early training in oral comprehension and phonological skills: results of a three-year longitudinal study ». Scientific Studies in Reading 14 (3), p. 211-246.

BONNÉRY, S. (2014). De l'étude des inégalités scolaire à celle de la domination scolaire. Pertinence de la dimension méso-sociologique en sociologie de l'école. Note de synthèse pour l'HDR. Université Paris 8. BRISSAUD, C. \& COGIS, D. (2011). Comment enseigner l'orthographe aujourd'hui ? Paris : Hatier.

BRISSAUD, C., VIRIOT-GOELDEL, C. \& PONTON, C. (2019). « Enseigner et apprendre l'orthographe avec la “Twictée”. Premiers résultats de l'évaluation d'un dispositif innovant d'enseignement de l'orthographe ». Repères 60, p. 107-130. En ligne : https://doi.org/10.4000/reperes.2346. 
CADET, L., CRINON, J. \& FERONE, G. (2019). « Former au raisonnement orthographique. Conceptions d'enseignants du cycle 3 ». Repères 60, p. 153-171. En ligne : https://doi.org/10.4000/reperes.2371. CÈBE, S. \& GOIGOUX, R. (2017). Narramus MS-GS. Paris : Retz.

CORDEIRO, G. S. \& SCHNEUWLY, B. (2007). « La construction de l'objet enseigné et les organisateurs du travail enseignant ». Recherche et formation 56, p. 67-80. En ligne : https://doi.org/10.4000/ rechercheformation.903.

CRINON, J. \& VIRIOT-GOELDEL, C. (2019). « Réflexions sur un dispositif pour enseigner et apprendre l'orthographe ». Communication au colloque de l'AIRDF-Association internationale de Recherche en Didactique du français. Lyon, 27-29 aout 2019.

DELARUE-BRETON, C. (2012). « Dispositifs et logique dispositive : perception des enjeux et inégalités scolaires ». In Élalouf, M.-L., Robert, A., Belhadjin, A. \& Bishop, M.-F. (dirs), Les Didactiques en question : état des lieux et perspectives pour la recherche et la formation. Bruxelles : De Boeck, p. $120-130$.

DEPP (Direction de l'évaluation, de la prospective et de la performance) (2016). « Les performances en orthographe des élèves en fin d'école primaire (1987-2007-2015) ». Note d'information 28. En ligne : https://www.education.gouv.fr/les-performances-en-orthographe-des-eleves-en-fin-decole-primaire-1987-2007-2015-1991.

ÉCALLE, J., GOMES, C., AUPHAN, P., CROS, L., \& MAGNAN, A. (2019). « Effects of policy and educational interventions intended to reduce difficulties in literacy skills in Grade 1 ». Studies in Educational Evaluation 61, p. 12-20. En ligne : https://doi.org/10.1016/j.stueduc.2019.02.001.

FENOGLIO, P. (2019). « Le hashtag pour catégoriser les erreurs d'orthographe au cycle 3 : un hiatus entre objectifs pédagogiques et appropriations des élèves ». Repères 60, p. 131-151. En ligne : https://doi.org/10.4000/reperes.2351. FOUCAULT, M. (1977). Dits et écrits, tome III. Paris : Gallimard.

GFEN (Groupe Français d'Éducation Nouvelle) (1982). Quelles pratiques pour une autre école ? Paris : Casterman.

GoIGoux, R. (dir.) (2020). À la manière de Narramus. Enquête sur la transposition de la méthode et son influence sur le développement professionnel des enseignants. Clermont-Ferrand : Université ClermontAuvergne, Inspé Clermont-Auvergne.

GOIGOUX, R., CÈBE, S. \& PAOUR, J.-L. (2004). Phono. Paris: Hatier.

HAAS, G. (1999). « Les ateliers de négociation graphique : une cadre de développement des compétences métalinguistiques pour des élèves de cycle 3 ». Repères 20, p. 127-142. En ligne : https://doi.org/10.3406/reper.1999.2315.

LAMBERT, É. \& DOYEN, A.-L. (2005). Phonoludos entrainement MS \& GS. Grenoble : Éditions de la Cigale.

LATOUR, в. (1996). « Les cornéliens dilemmes d'une ceinture de sécurité ». In Petites leçons de sociologie des sciences. Paris : Éditions La Découverte, p. 25-32.

REUTER, Y., COHEN-AZRIA, C., DAUNAY, B., DELCAMBRE, I. \& LAHANIER-REUTER, D. (2007). Dictionnaire des concepts fondamentaux de didactique. Villeneuve-d'Asq : Presses universitaires du Septentrion. VASQUEZ, A. \& OURY, F. (1967). Vers une pédagogie institutionnelle. Paris : F. Maspero.

VIRIOT-GOELDEL, C. (2011). « Les programmes "clé en main" aux États-Unis : des modèles d'intervention pédagogique en réponse à la crise de l'école ». Actes du colloque AECSE «Crise et/ en éducation ", 28-29 octobre 2011, UPO Nanterre La Défense. En ligne : https:// 
www.researchgate.net/publication/292988311_Les_programmes_cle_en_main_aux_EtatsUnis_des_modeles_d\%27intervention_pedagogique_en_reponse_a_la_crise_de_l\%27ecole.

\section{NOTES}

1. Voir : http://www.twictee.org/twictee/.

2. Il s'agit de la recherche "Twictée pour apprendre l'orthographe ", conduite dans le cadre du programme d'investissement d'avenir (PIA2), appel à projets e-FRAN.

3. Chaque classe est désignée par un numéro d'anonymat.

4. Nous désignons les enseignants par un numéro permettant de respecter leur anonymat.

5. Mais les variations de mises en œuvre pourraient être illustrées par bien d'autres exemples.

6. Ainsi certains programs mis en œuvre aux États-Unis sont brevetés, contractuels et adoptés dans le cadre d'une politique d'accountability, imposant un déroulement normé.

7. $M$ introduit les paroles du maitre et $E$ celles de l'élève.

8. Mot-clé utilisé dans les tweets, distingué par la présence d'un hashtag (\#). Dans le dispositif Twictée, les balises renvoient à une typologie de notions orthographiques.

\section{RÉSUMÉS}

Résumé : Un dispositif didactique implique la médiation par des instruments et un aménagement du milieu visant à obtenir des élèves un comportement déterminé. À partir de données issues de la recherche "Twictée pour apprendre l'orthographe" - mesure des progrès des élèves, entretiens avec des enseignants et observations de classes - nous montrons la confiance des enseignants dans les potentialités et les contraintes du dispositif pour enrôler et faire progresser les élèves, mais aussi l'importance de la qualité des interventions des enseignants, qui, dans le type de dispositif ici étudié, introduisent des variations considérables de sa mise en œuvre et modifient la nature du travail cognitif demandé aux élèves.

Abstract: The French term "dispositif didactique" describes a teaching intervention in which selected tools are applied within a specifically organized environment in order to help students attain one or more learning objectives. We used data (measures of students' progress, interviews with teachers, class observations) from a study of a new collaborative spelling intervention, called “Twictée", to examine teachers' appreciations of the intervention's potential for engaging students and enhancing their spelling performance. An analysis of class observations revealed considerable variations in the way teachers implemented the Twictée method that are likely to modify the nature of the cognitive work required. It also showed the importance of the quality of student-teacher interactions. 
INDEX

Keywords : collaborative learning, digital technologies, elementary school, interactions, language teaching, spelling instruction

Mots-clés : collaboration, enseignement de la langue, école élémentaire, interactions, numérique, orthographe

\section{AUTEURS}

JACQUES CRINON

Université Paris-Est Créteil, CIRCEFT, F-94380 Bonneuil, France

\section{CAROLINE VIRIOT-GOELDEL}

Université Paris-Est Créteil, CIRCEFT, F-94380 Bonneuil, France 